\title{
Exchange Algebra and the Drinfeld-Sokolov Theorem
}

\author{
O. Babelon ${ }^{1}$, F. Toppan ${ }^{1 \star}$ and L. Bonora ${ }^{2}$ \\ ${ }^{1}$ Laboratoire de Physique Théorique et Hautes Energies, Université Pierre et Marie Curie, Tour \\ $161^{\text {er }}$ Etage, 4 Place Jussieu, F-75252 Paris Cedex 05, France \\ ${ }^{2}$ International School for Advanced Studies (SISSA/ISAS), Strada Costiera 11, I-34014 Trieste, \\ Italy and INFN, Sezione di Trieste, I-34014 Trieste, Italy
}

Received August 14, 1990

\begin{abstract}
We analyse the relation between the exchange algebra and the separation of the chiralities in classical Toda field theory. We show that there exists a conformally covariant Bloch wave basis such that the two chiralities commute. In terms of this basis we then reconstruct the periodic and local solution of Toda field theory.
\end{abstract}

\section{Introduction}

Toda field theories are simultaneously conformal field theories and integrable models. A basic tool in the study of these theories is the exchange algebra [1,2]. Since we have a conformal theory, we expect the dynamics to split into a right moving sector and a left moving sector. However it so happens that the simplest choice for the exchange algebra coming from the integrable structure does not completely split the chiralities due to the zero mode problem. The aim of this paper is to analyse the relation between the exchange algebra and the separation of the chiralities in classical Toda field theory. In this first section we illustrate the problem more precisely, introduce the notation and outline the solution. The rest of the paper is devoted to the proofs of the various propositions.

Let $\mathscr{G}$ be a simple finite dimensional Lie algebra of rank $n$, equipped with an invariant scalar product denoted by (,). We choose a Cartan subalgebra $\mathscr{H}$ with an orthonormal basis $\left\{H_{i}\right\}$. We also need the Cartan decomposition of $\mathscr{G}$,

$$
\mathscr{G}=\mathscr{N}_{-} \oplus \mathscr{H} \oplus \mathscr{N}_{+} .
$$

We recall the following commutation relations in a Cartan-Weyl basis:

$$
\begin{aligned}
{\left[H, E_{ \pm \alpha}\right] } & = \pm \alpha(H) E_{ \pm \alpha}, \\
{\left[E_{\alpha}, E_{-\alpha}\right] } & =H_{\alpha} .
\end{aligned}
$$

* Work supported by Fondazione Angelo Della Riccia 
Toda field theories are defined by means of a linear system.

$$
\left(\partial_{z \pm}+A_{z \pm}\right) T=0
$$

where $z_{ \pm}=x \pm t$ are the light cone coordinates, $\partial_{z_{ \pm}}=\frac{1}{2}\left(\partial_{x} \pm \partial_{t}\right)$,

$$
\begin{aligned}
& A_{z_{+}}=\partial_{z_{+}} \Phi+e^{\operatorname{ad} \Phi} \mathscr{E}_{+}, \\
& A_{z_{-}}=-\partial_{z_{-}} \Phi+e^{-\operatorname{ad} \Phi} \mathscr{E}_{-} .
\end{aligned}
$$

The field $\Phi$ takes values in the Cartan subalgebra and

$$
\begin{aligned}
& \mathscr{E}_{+}=\sum_{\alpha \text { simple }} E_{\alpha}, \\
& \mathscr{E}_{-}=\sum_{\alpha \text { simple }} E_{-\alpha} .
\end{aligned}
$$

The zero curvature condition

$$
F_{z_{+} z_{-}}=\partial_{z_{+}} A_{z_{-}}-\partial_{z_{-}} A_{z_{+}}+\left[A_{z_{+}}, A_{z_{-}}\right]=0
$$

yields the equations of motion

$$
\partial_{z_{+}} \partial_{z_{-}} \Phi=\frac{1}{2} \sum_{\alpha \text { simple }} e^{2 \alpha(\Phi)} H_{\alpha} .
$$

Given a highest weight vector $\left|\lambda_{\max }^{(r)}\right\rangle$, we define

$$
\begin{aligned}
& \xi^{(r)}(x)=\left\langle\lambda_{\max }^{(r)}\right| e^{-\Phi(x)} T(x), \\
& \bar{\xi}^{(r)}(x)=T^{-1}(x) e^{-\Phi(x)}\left|\lambda_{\max }^{(r)}\right\rangle,
\end{aligned}
$$

where $T(x)$ is the transport matrix

$$
T(x)=P \exp \left(-\int_{0}^{x} A_{x} d x\right)
$$

and $A_{x}=A_{z_{+}}+A_{z_{-}}$. Notice that $T(x)$ is the solution of the equation $\left(\partial_{x}+A_{x}\right) T=0$ with boundary condition $T(0)=1$.

Using the explicit form of $A_{z_{+}}$and $A_{z_{-}}$one can easily show that $\xi$ and $\bar{\xi}$ are chiral objects, i.e.

$$
\partial_{z_{-}} \xi=0, \quad \partial_{z_{+}} \bar{\xi}=0
$$

The conformal structure of Toda field theory is encoded in the exchange algebra [2],

$$
\begin{aligned}
& \left\{\xi^{(r)}(x) \otimes \xi^{\left(r^{\prime}\right)}(y)\right\}=\xi^{(r)}(x) \otimes \xi^{\left(r^{\prime}\right)}(y)\left[\theta(x-y) r^{+}+\theta(y-x) r^{-}\right], \\
& \left\{\bar{\xi}^{(r)}(x) \otimes \bar{\xi}^{\left(r^{\prime}\right)}(y)\right\}=\left[\theta(x-y) r^{-}+\theta(y-x) r^{+}\right] \bar{\xi}^{(r)}(x) \otimes \bar{\xi}^{\left(r^{\prime}\right)}(y), \\
& \left\{\xi^{(r)}(x) \otimes \bar{\xi}^{\left(r^{\prime}\right)}(y)\right\}=-\xi^{(r)}(x) \otimes 1 \cdot r^{-} \cdot 1 \otimes \bar{\xi}^{\left(r^{\prime}\right)}(y), \\
& \left\{\bar{\xi}^{(r)}(x) \otimes \xi^{\left(r^{\prime}\right)}(y)\right\}=-1 \otimes \xi^{\left(r^{\prime}\right)}(y) \cdot r^{+} \cdot \bar{\xi}^{(r)}(x) \otimes 1,
\end{aligned}
$$

wher $r^{ \pm}$are the solutions of the classical Yang-Baxter equation given in Eq. $(31,32)$. If the Toda field $\Phi(x)$ is periodic, the $\xi, \bar{\xi}$ fields have the monodromy properties

$$
\begin{aligned}
& \xi^{(r)}(x+2 \pi)=\xi^{(r)}(x) T, \\
& \xi^{(r)}(x+2 \pi)=T^{-1} \bar{\xi}^{(r)}(x),
\end{aligned}
$$


where $T=T(2 \pi)$. We also recall the following Poisson brackets [3]:

$$
\begin{aligned}
& \{T \otimes T\}=-\left[r_{12}^{ \pm}, T \otimes T\right], \\
& \left\{\xi^{(r)}(x) \otimes T\right\}=\xi^{(r)}(x) \otimes T \cdot r^{-}, \\
& \left\{\bar{\xi}^{(r)}(x) \otimes, T\right\}=-1 \otimes T \cdot r^{+} \cdot \bar{\xi}^{(r)}(x) \otimes 1 .
\end{aligned}
$$

Finally, from Eq. (4), we see that the fields of Toda theory are reconstructed by means of the formula [4]

$$
e^{-2 \lambda_{\max }^{(r)}(\Phi)}=\xi^{(r)} \cdot \bar{\xi}^{(r)}
$$

These fields are obviously periodic and one can check that they are local. Equation (8) would yield a solution of the Toda field equation (3) if one could directly construct explicit realizations of Eqs. $(5,6,7)$ in terms of free fields.

Although $\xi$ and $\bar{\xi}$ are chiral objects and therefore split the dynamics into right and left moving sectors, there is a non-trivial coupling between them as can be seen from Eq. (5); this coupling can only occur through the zero modes and their conjugate variables. The purpose of this work is to disentangle this zero mode problem. The solution we propose will be obtained in two steps. First we construct a basis $\eta, \bar{\eta}$ with the following diagonal monodromy:

$$
\begin{aligned}
& \eta^{(r)}(x+2 \pi)=\eta^{(r)}(x) e^{2 \pi P_{0}}, \\
& \bar{\eta}^{(r)}(x+2 \pi)=e^{-2 \pi P_{0}} \bar{\eta}^{(r)}(x),
\end{aligned}
$$

and with the additional property

$$
\left\{\eta^{(r)}(x) \otimes \bar{\eta}^{\left(r^{\prime}\right)}(y)\right\}=0 .
$$

The full exchange algebra of $\eta, \bar{\eta}$ is given in Eqs. $(43,44)$. Moreover, we show that there exists a suitable diagonal matrix $\rho^{(r)}$ such that the local and periodic solutions of the Toda theory are given by

$$
e^{-2 \lambda_{\max }^{(r)}(\Phi)}=\eta^{(r)} \rho^{(r)} \bar{\eta}^{(r)}
$$

Equation (9) provides a complete solution to our problem of splitting the chiralities in Toda field theory. However it has a drawback. This appears when we analyse the conformal properties of the fields $\eta$ and $\bar{\eta}$. It is shown in Eq. (49) that they behave almost like covariant operators but not quite. We overcome this difficulty in the second step of our construction by enlarging the phase space. We consider the zero modes of the left and right movers, $P_{0}$ and $\bar{P}_{0}$ respectively, as independent dynamical variables. We also introduce their independent conjugate variables $q_{+}$ and $q_{-}$:

$$
\begin{aligned}
& \left\{P_{0}, q_{+}\right\}=\frac{1}{2 \pi}, \quad\left\{P_{0}, q_{-}\right\}=0 \\
& \left\{\bar{P}_{0}, q_{+}\right\}=0, \quad\left\{\bar{P}_{0}, q_{-}\right\}=\frac{1}{2 \pi} .
\end{aligned}
$$

Then we define

$$
\psi^{(r)}=\eta^{(r)} \rho_{+}^{(r)}, \quad \bar{\psi}^{(r)}=\rho_{-}^{(r)} \bar{\eta}^{(r)},
$$

where $\rho_{ \pm}^{(r)}$ are introduced in Eq. (24). The fields $\psi$ and $\bar{\psi}$ are covariant objects, 
they have diagonal monodromy

$$
\begin{aligned}
& \psi^{(r)}(x+2 \pi)=\psi^{(r)}(x) e^{2 \pi P_{0}} \\
& \bar{\psi}^{(r)}(x+2 \pi)=e^{-2 \pi \bar{P}_{0}} \bar{\psi}^{(r)}(x) .
\end{aligned}
$$

They satisfy

$$
\left\{\psi^{(r)}(x), \bar{\psi}^{\left(r^{\prime}\right)}(y)\right\}=0
$$

and their exchange algebra is given in Eq. $(51,52)$.

Moreover the expression

$$
e^{-2 \lambda_{\max }^{(r)}(\Phi)}=\psi^{(r)} \cdot \bar{\psi}^{(r)}
$$

admits a restriction to the original phase space, i.e. it has vanishing Poisson bracket with the constraint $P_{0}-\bar{P}_{0}=0$ :

$$
\left\{e^{-2 \lambda_{\max }^{(r)}(\Phi)}, P_{0}-\bar{P}_{0}\right\}=0 .
$$

Furthermore this restriction is periodic and local. It is therefore a satisfactory solution to our problem.

Let us now describe in more detail our construction. First we consider separately the two chiral halves of the theory. They are described by the so-called DrinfeldSokolov linear systems $[4,5]$

$$
\begin{aligned}
& \partial_{+} Q_{+}=\left(P-\mathscr{E}_{+}\right) Q_{+}, \\
& \partial_{-} Q_{-}=-Q_{-}\left(\bar{P}-\mathscr{E}_{-}\right),
\end{aligned}
$$

where $P$ and $\bar{P}$ are periodic fields which take values in the Cartan subalgebra and have the Poisson brackets

$$
\begin{aligned}
& \{P(x) \underset{\otimes}{\otimes} P(y)\}=\frac{1}{2}\left(\partial_{x}-\partial_{y}\right) \delta(x-y) \sum_{i} H_{i} \otimes H_{i}, \\
& \{P(x) \underset{\otimes}{\otimes}(y)\}=0, \\
& \{\bar{P}(x) \underset{,}{\otimes} \bar{P}(y)\}=-\frac{1}{2}\left(\partial_{x}-\partial_{y}\right) \delta(x-y) \sum_{i} H_{i} \otimes H_{i} .
\end{aligned}
$$

From the solution $Q_{+}(x)$ and $Q_{-}(x)$ of Eqs. $(14,15)$ normalised by $Q_{+}(0)=1, Q_{-}(0)=1$ we define a basis $\sigma, \bar{\sigma}$,

$$
\begin{aligned}
& \sigma^{(r)}(x)=\left\langle\lambda_{\max }^{(r)}\right| Q_{+}(x), \\
& \bar{\sigma}^{(r)}(x)=Q_{-}(x)\left|\lambda_{\max }^{(r)}\right\rangle .
\end{aligned}
$$

This basis has a Poisson bracket algebra similar to Eq. $(5)$, see Eqs. $(38,41)$, but with

$$
\left\{\sigma^{(r)}(x) \otimes, \bar{\sigma}^{\left(r^{\prime}\right)}(y)\right\}=0 .
$$

However $\sigma$ and $\bar{\sigma}$ still have a non-trivial monodromy

$$
\begin{aligned}
& \sigma^{(r)}(x+2 \pi)=\sigma^{(r)}(x) S, \\
& \bar{\sigma}^{(r)}(x+2 \pi)=\bar{S} \bar{\sigma}^{(r)}(x),
\end{aligned}
$$

where $S \in \exp \left(\mathscr{H}+\mathscr{N}_{+}\right)$is upper triangular, and $\bar{S} \in \exp \left(\mathscr{H}+\mathscr{N}_{-}\right)$is lower 
triangular. In the second step we diagonalize the matrices $S$ and $\bar{S}$,

$$
\begin{aligned}
& S=g^{-1} e^{2 \pi P_{0}} g, \\
& \bar{S}=\bar{g}^{-1} e^{-2 \pi P_{0}} \bar{g},
\end{aligned}
$$

where $g \in \exp \left(\mathscr{N}_{+}\right)$is strictly upper triangular and $\bar{g} \in \exp \left(\mathscr{N}_{-}\right)$is strictly lower triangular. Remark that $g$ and $\bar{g}$ are uniquely determined by these conditions. Next we define

$$
\begin{aligned}
& \eta^{(r)}(x)=\sigma^{(r)}(x) g^{-1}, \\
& \bar{\eta}^{(r)}(x)=\bar{g} \bar{\sigma}^{(r)}(x) .
\end{aligned}
$$

These quantities have a diagonal monodromy and they Poisson commute

$$
\left\{\eta^{(r)}(x) \otimes \bar{\eta}^{\left(r^{\prime}\right)}(y)\right\}=0
$$

because they do not depend on the conjugate variable of $P_{0}$. This variable is then introduced into the theory by means of the constant $\rho^{(r)}$ in Eq. (9):

$$
\rho^{(r)}=e^{-K+} \theta e^{K-}, \quad \theta=e^{q} .
$$

The constants $K_{ \pm}$are defined in Eqs. $(29,30)$; their role is to remove all explicit dependence on the normalisation point $x=0$. The diagonal matrix $q$ is the conjugate variable of $P_{0}$ :

$$
\left\{P_{0}, q\right\}=\frac{1}{2 \pi} \sum_{i} H_{i} \otimes H_{i} .
$$

Next we enlarge the phase space by considering the left and right zero modes $P_{0}$ and $\bar{P}_{0}$ as independent variables, together with their conjugate variables $q_{+}$ and $q_{-}$. We may write

$$
q=q_{+}+q_{-}
$$

The constants entering Eq. (11) are simply

$$
\rho_{+}^{(r)}=e^{-K_{+}} e^{q+}, \quad \rho_{-}^{(r)}=e^{q-} e^{K-} .
$$

The functions which admit a restriction to the original phase space are those depending only on $q_{+}+q_{-}$, as is the case for the field $e^{-\lambda_{\max }^{(r)}(\Phi)}$, see Eq. (13).

\section{The Drinfeld-Sokolov Linear System}

The Drinfeld-Sokolov linear system reads [5]

$$
\partial Q=\left(b-\mathscr{E}_{+}\right) Q
$$

where $b \in \mathscr{H} \oplus \mathscr{N}$.. The form of this system is invariant under the gauge transformations

$$
\begin{gathered}
{ }^{n} Q=n^{-1} Q \quad n \in \exp \left(\mathscr{N}_{-}\right), \\
{ }^{n} b-\mathscr{E}_{+}=n^{-1}\left(b-\mathscr{E}_{+}\right) n-n^{-1} \partial n .
\end{gathered}
$$

Choosing a gauge where $b=P \in \mathscr{H}$, we recover Eq. (14). For a derivation of Eq. (14) from Eqs. $(1,2)$, see [4]. 
We have already pointed out in the introduction that to reconstruct the Toda fields, we only need the projections of $Q_{+}(x)$ on highest weight vectors, Eq. (19). It is important to notice that the vectors $\sigma^{(r)}(x)$ are invariant under transformations, Eq. (26),

$$
\sigma^{(r)}(x)=\left\langle\lambda_{\max }^{(r)}\right| Q_{+}(x)=\left\langle\lambda_{\max }^{(r)}\right| n^{-1} Q_{+}(x)
$$

The matrix elements of the vector $\sigma^{(r)}(x)$ are just the first line of the matrix $Q(x)$ in the representation $\pi^{(r)}$ with highest weight $\lambda_{\max }^{(r)}$. From Eq. (25), we can write for these matrix elements a single higher order differential equation of order $\operatorname{dim} \pi^{(r)}$,

$$
\left[\sum_{i=0}^{\operatorname{dim} \pi^{(r)}} u_{i} \partial^{i}\right] \sigma^{(r)}(x)=0 .
$$

The coefficients $u_{i}(x)$ are local functions of $b$ and are invariant under Eq. (27) because the matrix elements of $\sigma^{(r)}(x)$ are themselves invariant. In fact they generate all such functions.

In principle, one should be able to work only with the invariant objects $\sigma^{(r)}(x)$ and Eq. $(28)[1,6]$. However the linear systems Eqs. $(25,14)$ are very powerful tools and we will use them systematically in this paper.

Our first task is to find the expression of the energy momentum tensor $\mathscr{U}=T_{++}$ in terms of the field $P$. Recall that the Poisson bracket algebra of the coefficients $u_{i}$ in Eq. (28) is known [5]. They generate a $W$-algebra which is the conformal symmetry algebra underlying the theory. In particular it contains the Virasoro algebra as a subalgebra. To construct its generator, we only have to look at local gauge invariant functions of $b$.

Proposition 2.1. Let $H_{\rho}$ be the element in $\mathscr{H}$ such that

$$
\left[H_{\rho}, \mathscr{E}_{+}\right]=\mathscr{E}_{+} \cdot
$$

Then the function

$$
\mathscr{U}=\frac{1}{2}\left(b-\mathscr{E}_{+}, b-\mathscr{E}_{+}\right)+\left(H_{\rho}, b^{\prime}\right)
$$

is invariant under the action Eq. (27).

Proof. It suffices to consider an infinitesimal transformation

$$
n=1+X, \quad X \in \mathscr{N}_{-} .
$$

We then have

$$
\delta b=\left[b-\mathscr{E}_{+}, X\right]-X^{\prime} .
$$

Using the invariance of $($,$) and the fact that \left(\mathscr{N}_{-}, \mathscr{N}_{-}\right)=0$ we get

$$
\delta \mathscr{U}=\left(\mathscr{E}_{+}, X^{\prime}\right)-\left(H_{\rho},\left[\mathscr{E}_{+}, X^{\prime}\right]\right) .
$$

Using the invariance again, the last term is

$$
\begin{aligned}
\left(H_{\rho},\left[\mathscr{E}_{+}, X^{\prime}\right]\right) & =\left(\left[H_{\rho}, \mathscr{E}_{+}\right], X^{\prime}\right) \\
& =\left(\mathscr{E}_{+}, X^{\prime}\right),
\end{aligned}
$$

which ends the proof. 
Evaluating the function $\mathscr{U}$ in the gauge where $b=P$ we find

$$
\mathscr{U}=\frac{1}{2}(P, P)+\left(H_{\rho}, P^{\prime}\right) .
$$

We recognize the Virasoro generator.

In the following we will be interested in the solution of Eq. (14) normalized to one at $x=0$,

$$
Q_{+}(0)=1 \text {. }
$$

Since $P(x)$ is a periodic field we introduce its Fourier expansion

$$
P(x)=\sum_{n} P_{n} e^{i n x},
$$

where $P_{n}$ take values in the Cartan subalgebra, and satisfy the Poisson brackets

$$
\left\{P_{n} \otimes P_{m}\right\}=\frac{i n}{2 \pi} \sum_{i} H_{i} \otimes H_{i} \delta_{n,-m} .
$$

The solution $Q_{+}(x)$ depends only on the field

$$
K_{+}(x)=\int_{0}^{x} d y P(y)
$$

We have

$$
K_{+}(x)=K_{+}+P_{0} x+\sum_{n \neq 0} \frac{P_{n}}{i n} e^{i n x},
$$

where the constant $K_{+}$is given by

$$
K_{+}=-\sum_{n \neq 0} \frac{P_{n}}{i n}
$$

In particular, we see that $Q_{+}(x)$ does not contain the variable $\theta$ conjugate to $P_{0}$. We have also

$$
\left\{P(x) \underset{\otimes}{\otimes} K_{+}\right\}=\left(\delta(x)-\frac{1}{2 \pi}\right) \sum_{i} H_{i} \otimes H_{i} .
$$

Another important object is the monodromy matrix

$$
Q_{+}(x+2 \pi)=Q_{+}(x) S .
$$

We have of course $S=Q_{+}(2 \pi)$. Notice that it is an upper triangular matrix. Its diagonal part is

$$
k=e^{2 \pi P_{0}} .
$$

It is convenient at this point to introduce the second chirality. As we have seen $Q_{-}$is characterized by the differential equation

$$
\partial Q_{-}=-Q_{-}\left(\bar{P}-\mathscr{E}_{-}\right)
$$

together with the boundary condition

$$
Q_{-}(0)=1
$$


We assume that $\bar{P}$ is periodic and takes values in the Cartan subalgebra,

$$
\bar{P}=\sum_{n} \bar{P}_{n} e^{i n x}
$$

We will impose

$$
\bar{P}_{0}=P_{0} .
$$

The matrix $Q_{-}$depends only on the field

$$
\begin{aligned}
K_{-}(x) & =K_{-}+P_{0} x+\sum_{n \neq 0} \frac{\bar{P}_{n}}{i n} e^{i n x}, \\
K_{-} & =-\sum_{n \neq 0} \frac{\bar{P}_{n}}{i n} .
\end{aligned}
$$

We will have

$$
Q_{-}(x+2 \pi)=\bar{S} Q_{-}(x)
$$

where $\bar{S}=Q_{-}(2 \pi)$. It is a lower triangular matrix, its diagonal part is $k^{-1}$. The Poisson bracket is defined by

$$
\left\{\bar{P}_{n} \otimes \bar{P}_{m}\right\}=-\frac{i n}{2 \pi} \sum_{i} H_{i} \otimes H_{i} \delta_{n,-m} .
$$

We have also

$$
\left\{\bar{P}(x) \otimes K_{-}\right\}=-\left(\delta(x)-\frac{1}{2 \pi}\right) \sum_{i} H_{i} \otimes H_{i}
$$

\section{Normalized Chiral Exchange Algebra}

Our aim is to compute the Poisson brackets $\left\{Q_{+}(x) \otimes Q_{+}(y)\right\},\left\{Q_{+}(x) \otimes S\right\}$ and $\{S \otimes S\}$ as well as the analogous objects for the other chirality. Using the definition Eq. (16) of the Poisson brackets we first prove a preliminary result.

\section{Lemma 3.1.}

$$
\begin{gathered}
\int_{0}^{x} d z \int_{0}^{y} d t \delta(z-t)\left(\partial_{t}-\partial_{z}\right) Q_{+}^{-1}(z) \otimes Q_{+}^{-1}(t)\left(\sum_{i} H_{i} \otimes H_{i}\right) Q_{+}(z) \otimes Q_{+}(t) \\
=\theta(x-y)\left\{-r+Q_{+}^{-1}(y) \otimes Q_{+}^{-1}(y) r Q_{+}(y) \otimes Q_{+}(y)\right\} \\
\quad+\theta(y-x)\left\{-r+Q_{+}^{-1}(x) \otimes Q_{+}^{-1}(x) r Q_{+}(x) \otimes Q_{+}(x)\right\},
\end{gathered}
$$

where $r$ is either $r^{+}$or $r^{-}$, the classical $r$-matrices of Toda field theory,

$$
\begin{aligned}
& r^{+}=\sum_{i} H_{i} \otimes H_{i}+2 \sum_{\alpha \text { positive }} \frac{E_{\alpha} \otimes E_{-\alpha}}{\left(E_{\alpha}, E_{-\alpha}\right)}, \\
& r^{-}=-\sum_{i} H_{i} \otimes H_{i}-2 \sum_{\alpha \text { positive }} \frac{E_{-\alpha} \otimes E_{\alpha}}{\left(E_{\alpha}, E_{-\alpha}\right)} .
\end{aligned}
$$


Proof. From Eq. (14) we get

$$
\begin{aligned}
\left(\partial_{t}\right. & \left.-\partial_{z}\right) Q_{+}^{-1}(z) \otimes Q_{+}^{-1}(t)\left(\sum_{i} H_{i} \otimes H_{i}\right) Q_{+}(z) \otimes Q_{+}(t) \\
& =Q_{+}^{-1}(z) \otimes Q_{+}^{-1}(t)\left(\sum_{i}\left[H_{i}, \mathscr{E}_{+}\right] \otimes H_{i}-H_{i} \otimes\left[H_{i}, \mathscr{E}_{+}\right]\right) Q_{+}(z) \otimes Q_{+}(t),
\end{aligned}
$$

and we are left with

$$
\begin{aligned}
& \int_{0}^{x} d z \int_{0}^{y} d t \delta(z-t)\left(\partial_{t}-\partial_{z}\right) Q_{+}^{-1}(z) \otimes Q_{+}^{-1}(t)\left(\sum_{i} H_{i} \otimes H_{i}\right) Q_{+}(z) \otimes Q_{+}(t) \\
& \quad=\int_{0}^{x} d z \theta(y-z) Q_{+}^{-1}(z) \otimes Q_{+}^{-1}(z)\left(\sum_{i}\left[H_{i}, \mathscr{E}_{+}\right] \otimes H_{i}-H_{i} \otimes\left[H_{i}, \mathscr{E}_{+}\right]\right) Q_{+}(z) \otimes Q_{+}(z) .
\end{aligned}
$$

But we have (see e.g. $[7,8]$ )

$$
\sum_{i}\left[H_{i}, \mathscr{E}_{+}\right] \otimes H_{i}-H_{i} \otimes\left[H_{i}, \mathscr{E}_{+}\right]=-\left[r, \mathscr{E}_{+} \otimes 1+1 \otimes \mathscr{E}_{+}\right],
$$

where $r$ is the classical $r$-matrix of Toda theory. As usual, $r$ can be either $r^{+}$or $r^{-}$. The result follows from the formula

$\left[r, Q_{+}(z) \otimes Q_{+}(z)\right]$

$$
=Q_{+}(z) \otimes Q_{+}(z) \int_{0}^{z} d t Q_{+}^{-1}(t) \otimes Q_{+}^{-1}(t)\left[r,\left(P-\mathscr{E}_{+}\right) \otimes 1+1 \otimes\left(P-\mathscr{E}_{+}\right)\right] Q_{+}(t) \otimes Q_{+}(t),
$$

where we have used that $r$ commutes with $H_{i} \otimes 1+1 \otimes H_{i}$.

Proposition 3.2. If $0<x, y<2 \pi$ we have

$\left\{Q_{+}(x) \otimes Q_{+}(y)\right\}$

$$
\begin{aligned}
= & \frac{1}{2} Q_{+}(x) \otimes Q_{+}(y)\left\{\theta(x-y)\left[-r+Q_{+}^{-1}(y) \otimes Q_{+}^{-1}(y)\left(r-\sum_{i} H_{i} \otimes H_{i}\right) Q_{+}(y) \otimes Q_{+}(y)\right]\right. \\
& \left.+\theta(y-x)\left[-r+Q_{+}^{-1}(x) \otimes Q_{+}^{-1}(x)\left(r+\sum_{i} H_{i} \otimes H_{i}\right) Q_{+}(x) \otimes Q_{+}(x)\right]\right\} .
\end{aligned}
$$

If $0<x<2 \pi$ and $y=2 \pi$ we have

$\left\{Q_{+}(x) \otimes, S\right\}=\frac{1}{2} Q_{+}(x) \otimes S$

$$
\left\{-r+Q_{+}^{-1}(x) \otimes Q_{+}^{-1}(x)\left(r+\sum_{i} H_{i} \otimes H_{i}\right) Q_{+}(x) \otimes Q_{+}(x)-\sum_{i} H_{i} \otimes S^{-1} H_{i} S\right\}
$$

If $x=2 \pi$ and $y=2 \pi$ we have

$$
\{S \otimes S\}=\frac{1}{2} S \otimes S\left\{-r+S^{-1} \otimes S^{-1} r S \otimes S+\sum_{i} S^{-1} H_{i} S \otimes H_{i}-H_{i} \otimes S^{-1} H_{i} S\right\} .
$$


Proof. Since $Q_{+}(x)$ is given by a path ordered exponential we may write

$$
\begin{aligned}
& \left\{Q_{+}(x) \otimes \underset{Q}{Q} Q_{+}(y)\right\} \\
& =Q_{+}(x) \otimes Q_{+}(y) \int_{0}^{x} d z \int_{0}^{y} d t Q_{+}^{-1}(z) \otimes Q_{+}^{-1}(t)\{P(z) \otimes, P(t)\} Q_{+}(z) \otimes Q_{+}(t) .
\end{aligned}
$$

Using Eq. (16), integrating by parts and writing all the terms explicitly we get

$$
\begin{aligned}
& \left\{Q_{+}(x) \otimes Q_{+}(y)\right\} \\
& =\frac{1}{2} Q_{+}(x) \otimes Q_{+}(y)\left\{\int_{0}^{y} d t \delta(x-t) Q_{+}^{-1}(x) \otimes Q_{+}^{-1}(t)\left(\sum_{i} H_{i} \otimes H_{i}\right) Q_{+}(x) \otimes Q_{+}(t)\right. \\
& \quad-\int_{0}^{y} d t \delta(t) \sum_{i} H_{i} \otimes Q_{+}^{-1}(t) H_{i} Q_{+}(t) \\
& \quad-\int_{0}^{x} d z \delta(z-y) Q_{+}^{-1}(z) \otimes Q_{+}^{-1}(y)\left(\sum_{i} H_{i} \otimes H_{i}\right) Q_{+}(z) \otimes Q_{+}(y) \\
& \quad+\int_{0}^{x} d z \delta(z) \sum_{i} Q_{+}^{-1}(z) H_{i} Q_{+}(z) \otimes H_{i} \\
& \left.\quad+\int_{0}^{x} d z \int_{0}^{y} d t \delta(z-t)\left(\partial_{t}-\partial_{z}\right) Q_{+}^{-1}(z) \otimes Q_{+}^{-1}(t)\left(\sum_{i} H_{i} \otimes H_{i}\right) Q_{+}(z) \otimes Q_{+}(t)\right\} .
\end{aligned}
$$

Since the field $P(x)$ is periodic, also the delta function in Eq. (16) is. So it is consistent to set

$$
\int_{0}^{y} d t \delta(t) f(t)=\left\{\begin{array}{lll}
\frac{1}{2} f(0) & \text { if } & 0<y<2 \pi \\
0 & \text { if } \quad y=0 \\
\frac{1}{2}(f(0)+f(2 \pi)) & \text { if } y=2 \pi
\end{array}\right.
$$

Using this definition, and the previous proposition we get the desired result. In Eq. (33) the symbol $\theta(0)$ must be understood as $\frac{1}{2}$.

The definition in Eq. (36) may look somewhat arbitrary. In fact one could introduce an arbitrary parameter $\alpha$ such that

$$
\int_{0}^{y} d t \delta(t) f(t)= \begin{cases}\alpha f(0) & \text { if } \quad 0<y<2 \pi \\ 0 & \text { if } y=0 \\ \alpha f(0)+(1-\alpha) f(2 \pi) & \text { if } y=2 \pi\end{cases}
$$

One easily sees that the results in Proposition 3.2 do not depend on $\alpha$. Only the intermediate results of Sects. 7, 8 and 9 depend on $\alpha$, but this dependence disappears in the results of all the other sections.

Define now

$$
\sigma^{(r)}(x)=\left\langle\lambda_{\max }^{(r)}\right| Q_{+}(x)
$$

Projecting out on highest weight vectors, we get $(0<x, y<2 \pi)$. 


\section{Corollary 3.3.}

$$
\begin{aligned}
& \left\{\sigma^{(r)}(x) \otimes \sigma^{\left(r^{\prime}\right)}(y)\right\}=-\frac{1}{2} \sigma^{(r)}(x) \otimes \sigma^{\left(r^{\prime}\right)}(y)\left\{r^{+} \theta(x-y)+r^{-} \theta(y-x)\right\}, \\
& \left\{\sigma^{(r)}(x) \otimes S\right\}=-\frac{1}{2}\left\{\sigma^{(r)}(x) \otimes S r^{-}+\sum_{i} \sigma^{(r)}(x) H_{i} \otimes H_{i} S\right\}, \\
& \{S \otimes, S\}=\frac{1}{2}\left\{[r, S \otimes S]+\sum_{i} H_{i} S \otimes S H_{i}-S H_{i} \otimes H_{i} S\right\} .
\end{aligned}
$$

Similarly, we define

$$
\bar{\sigma}^{(r)}(x)=Q_{-}(x)\left|\lambda_{\max }^{(r)}\right\rangle
$$

and get

\section{Corollary 3.4.}

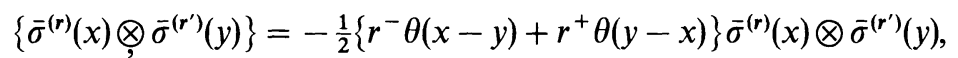

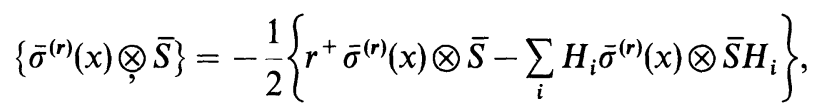

$$
\begin{aligned}
& \{\bar{S} \otimes, \bar{S}\}=-\frac{1}{2}\left\{[r, \bar{S} \otimes \bar{S}]+\sum_{i} \bar{S} H_{i} \otimes H_{i} \bar{S}-H_{i} \bar{S} \otimes \bar{S} H_{i}\right\} .
\end{aligned}
$$

\section{Poisson Brackets $\{g \underset{g}{\otimes} g$ \}}

As we have seen in the introduction, we need to diagonalize the triangular matrix $S$. This is done explicitly in the appendix, but we will not use in the following the detailed form of the diagonalization matrix $g$. We only need

$$
S=g^{-1} k g, \quad k=e^{2 \pi P_{0}} .
$$

Notice that $g$ is uniquely defined if we require it to be strictly upper triangular. We wish to compute the Poisson brackets of the matrix elements of $g$. Using that $k$ Poisson commutes with everything and inserting Eq. (42) into Eq. (40) we get

$$
\begin{aligned}
4 \operatorname{sh}( & \left.\pi \operatorname{ad}_{1} P_{0}\right) \operatorname{sh}\left(\pi \mathrm{ad}_{2} P_{0}\right)\{g \otimes g\} g^{-1} \otimes g^{-1} \\
= & \frac{1}{2}\left\{-2 \operatorname{sh}\left(\pi \operatorname{ad}_{1} P_{0}+\pi \operatorname{ad}_{2} P_{0}\right) g \otimes g r g^{-1} \otimes g^{-1}\right. \\
& \left.\quad-2 \operatorname{sh}\left(\pi \operatorname{ad}_{1} P_{0}-\pi \operatorname{ad}_{2} P_{0}\right) \sum_{i} g \otimes g H_{i} \otimes H_{i} g^{-1} \otimes g^{-1}\right\},
\end{aligned}
$$

where

Since

$$
\operatorname{ad}_{1} P_{0}=\operatorname{ad} P_{0} \otimes 1, \quad \operatorname{ad}_{2} P_{0}=1 \otimes \operatorname{ad} P_{0} .
$$

$$
\begin{aligned}
\left(\operatorname{ad}_{1} P_{0}+\operatorname{ad}_{2} P_{0}\right) r & =0, \\
\left(\operatorname{ad}_{1} P_{0}-\operatorname{ad}_{2} P_{0}\right) H_{i} \otimes H_{i} & =0,
\end{aligned}
$$


we may write also

$\operatorname{sh}\left(\pi \operatorname{ad}_{1} P_{0}\right) \operatorname{sh}\left(\pi \operatorname{ad}_{2} P_{0}\right)\{g \otimes g\} g^{-1} \otimes g^{-1}$

$$
\begin{aligned}
= & \left.-\frac{1}{4} \operatorname{sh}\left(\pi \operatorname{ad}_{1} P_{0}\right) \operatorname{ch}\left(\pi \operatorname{ad}_{2} P_{0}\right)\left[g \otimes g\left(r+\sum_{i} H_{i} \otimes H_{i}\right) g^{-1} \otimes g^{-1}-r-\sum_{i} H_{i} \otimes H_{i}\right)\right] \\
& \left.-\frac{1}{4} \operatorname{sh}\left(\pi \operatorname{ad}_{2} P_{0}\right) \operatorname{ch}\left(\pi \operatorname{ad}_{1} P_{0}\right)\left[g \otimes g\left(r-\sum_{i} H_{i} \otimes H_{i}\right) g^{-1} \otimes g^{-1}-r+\sum_{i} H_{i} \otimes H_{i}\right)\right] .
\end{aligned}
$$

This equation determines $\{g \otimes g\} g^{-1} \otimes g^{-1}$ since this matrix is in $\mathscr{N}_{+} \otimes \mathscr{N}_{+}$and the set

$$
\operatorname{Ker} \operatorname{sh}\left(\pi \operatorname{ad}_{1} P_{0}\right) \operatorname{sh}\left(\pi \operatorname{ad}_{2} P_{0}\right)=\mathscr{H} \otimes \mathscr{G}+\mathscr{G} \otimes \mathscr{H}
$$

has no component on $\mathscr{N}_{+} \otimes \mathscr{N}_{+}$. So we find modulo $\operatorname{Ker} \operatorname{sh}\left(\pi \operatorname{ad}_{1} P_{0}\right) \operatorname{sh}\left(\pi \operatorname{ad}_{2} P_{0}\right)$,

$$
\begin{aligned}
\{g \otimes, g\} g^{-1} \otimes g^{-1} & \\
= & \left.-\frac{1}{4} \operatorname{coth}\left(\pi \operatorname{ad}_{2} P_{0}\right)\left[g \otimes g\left(r+\sum_{i} H_{i} \otimes H_{i}\right) g^{-1} \otimes g^{-1}-r-\sum_{i} H_{i} \otimes H_{i}\right)\right] \\
& \left.-\frac{1}{4} \operatorname{coth}\left(\pi \operatorname{ad}_{1} P_{0}\right)\left[g \otimes g\left(r-\sum_{i} H_{i} \otimes H_{i}\right) g^{-1} \otimes g^{-1}-r+\sum_{i} H_{i} \otimes H_{i}\right)\right] .
\end{aligned}
$$

The element of $\operatorname{Ker} \operatorname{sh}\left(\pi \operatorname{ad}_{1} P_{0}\right) \operatorname{sh}\left(\pi \operatorname{ad}_{2} P_{0}\right)$ to be added is determined by requiring the right-hand side of this equation to be in $\mathscr{N}_{+} \otimes \mathscr{N}_{+}$. Let us consider the expression

$$
E=g \otimes g\left(r+\sum_{i} H_{i} \otimes H_{i}\right) g^{-1} \otimes g^{-1}-r-\sum_{i} H_{i} \otimes H_{i} .
$$

In this expression $r$ can be either $r^{+}$or $r^{-}$. Setting $r=r^{-}$we see that $E \in \mathscr{G} \otimes \mathscr{N}_{+}$ In particular, this shows that the action of $\operatorname{coth}\left(\pi \mathrm{ad}_{2} P_{0}\right)$ is well defined on $E$ since ad $P_{0}$ does not vanish on $\mathscr{N}_{+}$. Setting $r=r^{+}$we see that $E \in \mathscr{B}_{+} \otimes \mathscr{G}$, where $\mathscr{B}_{+}=\mathscr{H} \oplus \mathscr{N}_{+}$. The component of $E$ on $\mathscr{H} \otimes \mathscr{G}$ is equal to

$$
2 \sum_{i}\left(H_{i} \otimes g H_{i} g^{-1}-H_{i} \otimes H_{i}\right) \in \mathscr{H} \otimes \mathscr{N}_{+} \cdot
$$

This is indeed in $\operatorname{Ker} \operatorname{sh}\left(\pi \operatorname{ad}_{1} P_{0}\right) \operatorname{sh}\left(\pi \operatorname{ad}_{2} P_{0}\right)$. We must subtract this term to obtain something in $\mathscr{N}_{+} \otimes \mathscr{N}_{+}$. Proceeding in an analogous way for the other term, we finally get

\section{Proposition 4.1.}

$$
\begin{aligned}
\{g \otimes g\} g^{-1} \otimes g^{-1}= & -\frac{1}{2} \operatorname{coth}\left(\pi \operatorname{ad}_{2} P_{0}\right)\left[g \otimes g\left(r+\sum_{i} H_{i} \otimes H_{i}\right) g^{-1} \otimes g^{-1}-r\right. \\
& \left.-\sum_{i} H_{i} \otimes H_{i}-2 \sum_{i}\left(H_{i} \otimes g H_{i} g^{-1}-H_{i} \otimes H_{i}\right)\right] \\
& -\frac{1}{4} \operatorname{coth}\left(\pi \operatorname{ad}_{1} P_{0}\right)\left[g \otimes g\left(r-\sum_{i} H_{i} \otimes H_{i}\right) g^{-1} \otimes g^{-1}-r\right. \\
& \left.+\sum_{i} H_{i} \otimes H_{i}+2 \sum_{i}\left(g H_{i} g^{-1} \otimes H_{i}-H_{i} \otimes H_{i}\right)\right] .
\end{aligned}
$$


Similarly, setting

we obtain

$$
\bar{S}=\bar{g}^{-1} k^{-1} \bar{g}
$$

\section{Proposition 4.2.}

$$
\begin{aligned}
\{\bar{g} \otimes, \bar{g}\} \bar{g}^{-1} \otimes \bar{g}^{-1}= & -\frac{1}{4} \operatorname{coth}\left(\pi \operatorname{ad}_{2} P_{0}\right)\left[\bar{g} \otimes \bar{g}\left(r-\sum_{i} H_{i} \otimes H_{i}\right) \bar{g}^{-1} \otimes \bar{g}^{-1}-r\right. \\
& \left.+\sum_{i} H_{i} \otimes H_{i}+2 \sum_{i}\left(H_{i} \otimes \bar{g} H_{i} \bar{g}^{-1}-H_{i} \otimes H_{i}\right)\right] \\
& -\frac{1}{4} \operatorname{coth}\left(\pi \operatorname{ad}_{1} P_{0}\right)\left[\bar{g} \otimes \bar{g}\left(r+\sum_{i} H_{i} \otimes H_{i}\right) \bar{g}^{-1} \otimes \bar{g}^{-1}-r\right. \\
& \left.-\sum_{i} H_{i} \otimes H_{i}-2 \sum_{i}\left(\bar{g} H_{i} \bar{g}^{-1} \otimes H_{i}-H_{i} \otimes H_{i}\right)\right] .
\end{aligned}
$$

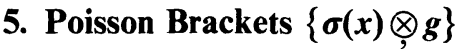

With the same method as in the previous section, starting from Eq. (39), we get modulo $\operatorname{Kerad}_{2} P_{0}$,

$$
\begin{aligned}
\{\sigma(x) \otimes g\} 1 \otimes g^{-1}= & -\frac{1}{4} \operatorname{coth}\left(\pi \operatorname{ad}_{2} P_{0}\right) \sigma(x) \otimes g\left(r^{-}+\sum_{i} H_{i} \otimes H_{i}\right) 1 \otimes g^{-1} \\
& -\frac{1}{4} \sigma(x) \otimes g\left(r^{-}-\sum_{i} H_{i} \otimes H_{i}\right) 1 \otimes g^{-1} .
\end{aligned}
$$

The result must be in $X \otimes \mathscr{N}_{+}$. The first term is all right. The second term is in $X \otimes \mathscr{B}_{+}$. Its component in $\operatorname{Ker}^{a_{2}} P_{0}=X \otimes \mathscr{H}$ is

$$
\frac{1}{2} \sum_{i} \sigma(x) H_{i} \otimes H_{i}
$$

we must substract this term. Thus we have proved

\section{Proposition 5.1.}

$$
\begin{aligned}
\{\sigma(x) \otimes g\} 1 \otimes g^{-1}= & -\frac{1}{4} \operatorname{coth}\left(\pi \operatorname{ad}_{2} P_{0}\right) \sigma(x) \otimes g\left(r^{-}+\sum_{i} H_{i} \otimes H_{i}\right) 1 \otimes g^{-1} \\
& -\frac{1}{4} \sigma(x) \otimes g\left(r^{-}-\sum_{i} H_{i} \otimes H_{i}\right) 1 \otimes g^{-1}-\frac{1}{2} \sum_{i} \sigma(x) H_{i} \otimes H_{i} .
\end{aligned}
$$

For the other chirality, we find

\section{Proposition 5.2.}

$$
\begin{aligned}
\{\bar{\sigma}(x) \otimes, \bar{g}\} 1 \otimes \bar{g}^{-1}= & \frac{1}{4} \operatorname{coth}\left(\pi \operatorname{ad}_{2} P_{0}\right)\left[1 \otimes \bar{g}\left(r^{+}-\sum_{i} H_{i} \otimes H_{i}\right) \bar{\sigma}(x) \otimes \bar{g}^{-1}\right] \\
& +\frac{1}{4} 1 \otimes \bar{g}\left(r^{+}+\sum_{i} H_{i} \otimes H_{i}\right) \bar{\sigma}(x) \otimes \bar{g}^{-1}-\frac{1}{2} \sum_{i} H_{i} \bar{\sigma}(x) \otimes H_{i} .
\end{aligned}
$$




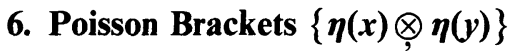

Let us define

$$
\eta(x)=\sigma(x) g^{-1} .
$$

As a consequence of the definition of $g$, this $\eta$ basis has already a diagonal monodromy

$$
\eta(x+2 \pi)=\eta(x) k \text {. }
$$

Using the previous results it is straightforward to derive the following

\section{Proposition 6.1.}

$$
\begin{aligned}
\{\eta(x) \otimes, \eta(y)\}= & -\frac{1}{4} \eta(x) \otimes \eta(y)\left(r^{+}-r^{-}\right) \varepsilon(x-y) \\
& +\frac{1}{4} \eta(x) \otimes \eta(y)\left\{\operatorname{coth}\left(\pi \operatorname{ad}_{1} P_{0}\right)\left[r^{+}-\sum_{i} H_{i} \otimes H_{i}\right]\right. \\
& \left.+\operatorname{coth}\left(\pi \operatorname{ad}_{2} P_{0}\right)\left[r^{-}+\sum_{i} H_{i} \otimes H_{i}\right]\right\} \\
& -\frac{1}{2} \eta(x) \otimes \eta(y)\left\{\left[\operatorname{coth}\left(\pi \operatorname{ad}_{1} P_{0}\right)-1\right] \sum_{i}\left(g H_{i} g^{-1} \otimes H_{i}-H_{i} \otimes H_{i}\right)\right. \\
& \left.-\left[\operatorname{coth}\left(\pi \operatorname{ad}_{2} P_{0}\right)-1\right] \sum_{i}\left(H_{i} \otimes g H_{i} g^{-1}-H_{i} \otimes H_{i}\right)\right\}
\end{aligned}
$$

We have used that

$$
g \otimes g\left(r^{+}-r^{-}\right) g^{-1} \otimes g^{-1}=r^{+}-r^{-} .
$$

Next, defining

$$
\bar{\eta}(x)=\bar{g} \bar{\sigma}(x)
$$

we get in an analogous way

\section{Proposition 6.2.}

$$
\begin{aligned}
\{\bar{\eta}(x) \otimes \bar{\eta}(y)\}= & \frac{1}{4}\left(r^{+}-r^{-}\right) \varepsilon(x-y) \bar{\eta}(x) \otimes \bar{\eta}(y)+\frac{1}{4}\left\{\operatorname{coth}\left(\pi \operatorname{ad}_{1} P_{0}\right)\left[r^{-}+\sum_{i} H_{i} \otimes H_{i}\right]\right. \\
& \left.+\operatorname{coth}\left(\pi \operatorname{ad}_{2} P_{0}\right)\left[r^{+}-\sum_{i} H_{i} \otimes H_{i}\right]\right\} \bar{\eta}(x) \otimes \bar{\eta}(y) \\
& +\frac{1}{2}\left\{\left[\operatorname{coth}\left(\pi \operatorname{ad}_{1} P_{0}\right)-1\right] \sum_{i}\left(\bar{g} H_{i} \bar{g}^{-1} \otimes H_{i}-H_{i} \otimes H_{i}\right)\right. \\
& \left.-\left[\operatorname{coth}\left(\pi \operatorname{ad}_{2} P_{0}\right)-1\right] \sum_{i}\left(H_{i} \otimes \bar{g} H_{i} \bar{g}^{-1}-H_{i} \otimes H_{i}\right)\right\} \bar{\eta}(x) \otimes \bar{\eta}(y) .
\end{aligned}
$$


Remark. In this Bloch wave type basis the two chiralities commute

$$
\{\eta(x) \otimes ฺ \bar{\eta}(y)\}=0 \text {. }
$$

\section{The Constant $\rho$}

Following our experience with Liouville we set

$$
\rho=e^{-K+} e^{K}-\theta
$$

where $K_{+}$is the constant defined in Eq. (29) and $K_{-}$is defined in Eq. (30). The constant $\theta$ is the conjugate variable of $P_{0}$,

$$
\begin{aligned}
\left\{\theta \otimes \bigotimes_{0}\right\} & =\frac{1}{2 \pi} \sum_{i} \theta H_{i} \otimes H_{i}, \\
\{\rho \otimes, k\} & =\rho \otimes k \sum_{i} H_{i} \otimes H_{i}, \\
\{\rho \otimes, \rho\} & =0 .
\end{aligned}
$$

Then we have

$$
\begin{aligned}
\left\{Q_{+}(x) \otimes e^{-K+}\right\}= & -Q_{+}(x) \otimes e^{-K_{+}} \int_{0}^{x} d z \delta(z) Q_{+}^{-1}(z) H_{i} Q_{+}(z) \otimes H_{i} \\
& +\frac{1}{2 \pi} Q_{+}(x) \otimes e^{-K_{+}} \int_{0}^{x} d z Q_{+}^{-1}(z) H_{i} Q_{+}(z) \otimes H_{i}
\end{aligned}
$$

and also

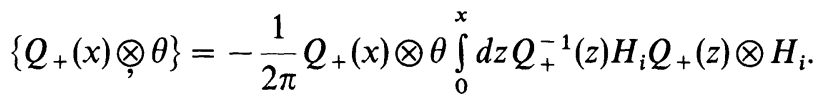

Therefore

$$
\left\{Q_{+}(x) \underset{,}{\otimes} \rho\right\}=-Q_{+}(x) \otimes \rho \int_{0}^{x} d z \delta(z) Q_{+}^{-1}(z) H_{i} Q_{+}(z) \otimes H_{i},
$$

so that finally we find

\section{Proposition 7.1.}

$$
\begin{aligned}
\{\sigma(x) \otimes, \rho\} & =-\frac{1}{2} \sigma(x) \otimes \rho \sum_{i} H_{i} \otimes H_{i}, \\
\{S \otimes \rho & =-\frac{1}{2} S \otimes \rho \sum_{i}\left[H_{i} \otimes H_{i}+S^{-1} H_{i} S \otimes H_{i}\right] .
\end{aligned}
$$

Similarly for the other chirality, we have

\section{Proposition 7.2.}

$$
\begin{aligned}
\{\bar{\sigma}(x) \otimes, \rho\} & =\frac{1}{2}\left(\sum_{i} H_{i} \otimes H_{i}\right) \bar{\sigma}(x) \otimes \rho, \\
\{\bar{S} \otimes \rho\} & =\frac{1}{2} \sum_{i}\left[H_{i} \otimes H_{i}+\bar{S} H_{i} \bar{S}^{-1} \otimes H_{i}\right] \bar{S} \otimes \rho .
\end{aligned}
$$




\section{Poisson Brackets $\{g \underset{, \rho}{\otimes}\}$}

We start from

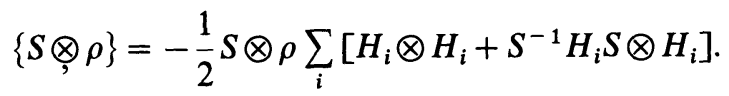

Setting

we find

$$
S=g^{-1} k g,
$$

$$
2 \operatorname{sh}\left(\pi \operatorname{ad}_{1} P_{0}\right)\left\{g \otimes \rho \rho g^{-1} \otimes 1-\sum_{i} H_{i} \otimes \rho H_{i}=-\operatorname{ch}\left(\pi \operatorname{ad}_{1} P_{0}\right) \sum_{i} g H_{i} g^{-1} \otimes \rho H_{i},\right.
$$

so that

\section{Proposition 8.1.}

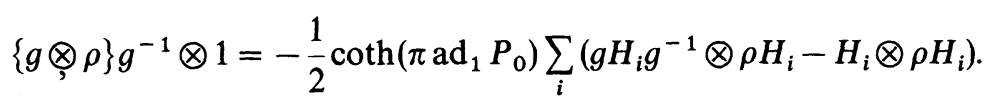

For the other chirality we have similarly

$$
\left\{\bar{g} \otimes \rho \rho \bar{g}^{-1} \otimes 1=-\frac{1}{2} \operatorname{coth}\left(\pi \operatorname{ad}_{1} P_{0}\right) \sum_{i}\left(\bar{g} H_{i} \bar{g}^{-1} \otimes \rho H_{i}-H_{i} \otimes \rho H_{i}\right) .\right.
$$

\section{Poisson Brackets $\{\eta(x) \otimes \rho, \rho\}$}

Recall that

$$
\begin{aligned}
& \eta(x)=\sigma(x) g^{-1}, \\
& \bar{\eta}(x)=\bar{g} \bar{\sigma}(x) .
\end{aligned}
$$

Then we find

\section{Proposition 9.1.}

$$
\begin{aligned}
\{\eta(x) \otimes \rho \rho\}= & -\frac{1}{2} \eta(x) \otimes \rho \sum_{i} g H_{i} g^{-1} \otimes H_{i} \\
& +\frac{1}{2} \eta(x) \otimes \rho \sum_{i} \operatorname{coth}\left(\pi \operatorname{ad}_{1} P_{0}\right)\left[g H_{i} g^{-1} \otimes H_{i}-H_{i} \otimes H_{i}\right], \\
\{\bar{\eta}(x) \otimes, \rho\}= & \frac{1}{2}\left(\sum_{i} \bar{g} H_{i} \bar{g}^{-1} \otimes H_{i}\right) \bar{\eta}(x) \otimes \rho \\
& -\frac{1}{2} \sum_{i} \operatorname{coth}\left(\pi \operatorname{ad}_{1} P_{0}\right)\left[\bar{g} H_{i} \bar{g}^{-1} \otimes H_{i}-H_{i} \otimes H_{i}\right] \bar{\eta}(x) \otimes \rho .
\end{aligned}
$$

\section{Application to Toda Field Theory}

Theorem 10.1. The fields

$$
e^{-2 \lambda^{(r)}(\Phi)}=\eta^{(r)} \rho^{(r)} \bar{\eta}^{(r)}
$$


1) Satisfy the Toda field equations,

2) are periodic,

3) are local.

\section{Proof.}

1) Recall the relation

$$
\alpha\left(H_{\alpha}\right)=(\alpha, \alpha) \text {. }
$$

The fundamental highest weights satisfy

$$
\lambda^{(i)}\left(H_{\alpha_{j}}\right)=\frac{1}{2}\left(\alpha_{i}, \alpha_{i}\right) \delta_{i j}
$$

Using this property, the equations of motion of Toda field theory, Eq. (3), become

$$
\partial_{z_{+}} \partial_{z_{-}} \lambda^{(i)}(\Phi)=\frac{1}{4}\left(\alpha_{i}, \alpha_{i}\right) e^{2 \alpha_{i}(\Phi)} .
$$

It is well known that

$$
\alpha_{i}=\sum_{j} 2 \frac{\left(\alpha_{i}, \alpha_{j}\right)}{\left(\alpha_{j}, \alpha_{j}\right)} \lambda^{(j)}=\sum_{j} a_{i j} \lambda^{(j)}
$$

where $a_{i j}$ is the Cartan matrix. Therefore

$$
\alpha_{i}(\Phi)=\sum_{j} a_{i j} \lambda^{(j)}(\Phi)
$$

and the equations of motion become

$$
\partial_{z_{+}} \partial_{z_{-}} \lambda^{(i)}(\Phi)=\frac{1}{4}\left(\alpha_{i}, \alpha_{i}\right) \prod_{j}\left[e^{-2 \lambda^{(j)}(\Phi)}\right]^{-a_{i j}}
$$

Our reconstruction formula Eq. (46) may be written

or

$$
e^{-2 \lambda^{(i)}(\Phi)}=\left\langle\lambda^{(i)}\left|Q_{+} g^{-1} \rho \bar{g} Q_{-}\right| \lambda^{(i)}\right\rangle
$$

$$
\lambda^{(i)}(\Phi)=-\frac{1}{2} \log \eta^{(i)} \rho \bar{\eta}^{(i)}
$$

It follows that

$$
\partial_{z_{+}} \partial_{z_{-}} \lambda^{(i)}(\Phi)=-\frac{1}{2} \frac{\operatorname{det}\left(\begin{array}{cc}
\eta^{(i)} \rho \bar{\eta}^{(i)} & \eta^{(i)} \rho \partial \bar{\eta}^{(i)} \\
\partial \eta^{(i)} \rho \bar{\eta}^{(i)} & \partial \eta^{(i)} \rho \partial \bar{\eta}^{(i)}
\end{array}\right)}{\left(\eta^{(i)} \rho \bar{\eta}^{(i)}\right)^{2}}
$$

Checking the equations of motion thus reduces to proving

$$
\operatorname{det}\left(\begin{array}{cc}
\eta^{(i)} \rho \bar{\eta}^{(i)} & \eta^{(i)} \rho \partial \bar{\eta}^{(i)} \\
\partial \eta^{(i)} \rho \bar{\eta}^{(i)} & \partial \eta^{(i)} \rho \partial \bar{\eta}^{(i)}
\end{array}\right)=-\frac{1}{2} \alpha_{i}^{2} \prod_{j \neq i}\left[e^{-2 \lambda^{(j)}(\Phi)} \cdot\right]^{-a_{i j}}
$$

This relation should be a consequence of the equations we have on $Q_{ \pm}$, Eq. $(14,15)$. Let us set

then

$$
Q_{+} \rho Q_{-}=G
$$

$$
\begin{aligned}
\operatorname{det}\left(\begin{array}{cc}
\eta^{(i)} \rho \bar{\eta}^{(i)} & \eta^{(i)} \rho \partial \bar{\eta}^{(i)} \\
\partial \eta^{(i)} \rho \bar{\eta}^{(i)} & \partial \eta^{(i)} \rho \partial \bar{\eta}^{(i)}
\end{array}\right)= & -\left\langle\lambda^{(i)}|G| \lambda^{(i)}\right\rangle\left\langle\lambda^{(i)}\left|\mathscr{E}_{+} G \mathscr{E}_{-}\right| \lambda^{(i)}\right\rangle \\
& +\left\langle\lambda^{(i)}\left|\mathscr{E}_{+} G\right| \lambda^{(i)}\right\rangle\left\langle\lambda^{(i)}\left|G \mathscr{E}_{-}\right| \lambda^{(i)}\right\rangle .
\end{aligned}
$$


But this can be rewritten as

where

$$
\operatorname{det}\left(\begin{array}{cc}
\eta^{(i)} \rho \bar{\eta}^{(i)} & \eta^{(i)} \rho \partial \bar{\eta}^{(i)} \\
\partial \eta^{(i)} \rho \bar{\eta}^{(i)} & \partial \eta^{(i)} \rho \partial \bar{\eta}^{(i)}
\end{array}\right)=-\frac{1}{2}\left\langle\Psi^{(i)}|G \otimes G| \Psi^{(i)}\right\rangle
$$

$$
\left|\Psi^{(i)}\right\rangle=\left|\lambda^{(i)}\right\rangle \otimes \mathscr{E}_{-}\left|\lambda^{(i)}\right\rangle-\mathscr{E}_{-}\left|\lambda^{(i)}\right\rangle \otimes\left|\lambda^{(i)}\right\rangle .
$$

Now the vectors $\left|\Psi^{(i)}\right\rangle$ are highest weight vectors. To see this we must show that

$$
\left(E_{\alpha} \otimes I+I \otimes E_{\alpha}\right)\left|\Psi^{(i)}\right\rangle=0 \quad \forall \alpha \text { positive. }
$$

It is enough to consider $\alpha$ simple. We have

$$
\left(E_{\alpha} \otimes I+I \otimes E_{\alpha}\right)\left|\Psi^{(i)}\right\rangle=\left|\lambda^{(i)}\right\rangle \otimes E_{\alpha} \mathscr{E}_{-}\left|\lambda^{(i)}\right\rangle-E_{\alpha} \mathscr{E}_{-}\left|\lambda^{(i)}\right\rangle \otimes\left|\lambda^{(i)}\right\rangle .
$$

But

$$
E_{\alpha} \mathscr{E}_{-}\left|\lambda^{(i)}\right\rangle=\lambda^{(i)}\left(H_{\alpha}\right)\left|\lambda^{(i)}\right\rangle
$$

and the statement follows.

The next step is to show that

$$
\left|\Psi^{(i)}\right\rangle=\left(\alpha_{i}, \alpha_{i}\right)^{1 / 2} \bigotimes_{j \neq i}\left(\left|\lambda^{(j)}\right\rangle\right)^{\otimes-a_{i j}}
$$

To see this we must prove that $\left|\Psi^{(i)}\right\rangle$ is an eigenstate of $H_{\alpha} \otimes I+I \otimes H_{\alpha}$ and find the eigenvalue. We have

$$
\begin{aligned}
\left(H_{\alpha} \otimes I+I \otimes H_{\alpha}\right)\left|\Psi^{(i)}\right\rangle= & H_{\alpha}\left|\lambda^{(i)}\right\rangle \otimes \mathscr{E}_{-}\left|\lambda^{(i)}\right\rangle-H_{\alpha} \mathscr{E}_{-}\left|\lambda^{(i)}\right\rangle \otimes\left|\lambda^{(i)}\right\rangle \\
& +\left|\lambda^{(i)}\right\rangle \otimes H_{\alpha} \mathscr{E}_{-}\left|\lambda^{(i)}\right\rangle-\mathscr{E}_{-}\left|\lambda^{(i)}\right\rangle \otimes H_{\alpha}\left|\lambda^{(i)}\right\rangle .
\end{aligned}
$$

Next we notice that

$$
\mathscr{E}_{-}\left|\lambda^{(i)}\right\rangle=E_{-\alpha_{l}}\left|\lambda^{(i)}\right\rangle .
$$

This may be seen by computing the norm of the vector $E_{-\alpha}\left|\lambda^{(i)}\right\rangle$. We find

$$
\left\langle\lambda^{(i)}\left|E_{\alpha} E_{-\alpha}\right| \lambda^{(i)}\right\rangle=\lambda^{(i)}\left(H_{\alpha}\right)
$$

which vanishes if $\alpha$ is not equal to $\alpha_{i}$. Therefore

$$
\left|\Psi^{(i)}\right\rangle=\left|\lambda^{(i)}\right\rangle \otimes E_{-\alpha_{i}}\left|\lambda^{(i)}\right\rangle-E_{-\alpha_{i}}\left|\lambda^{(i)}\right\rangle \otimes\left|\lambda^{(i)}\right\rangle .
$$

Using this remark, we obtain

$$
\left(H_{\alpha} \otimes I+I \otimes H_{\alpha}\right)\left|\Psi^{(i)}\right\rangle=\left[2 \lambda^{(i)}\left(H_{\alpha}\right)-\alpha_{i}\left(H_{\alpha}\right)\right]\left|\Psi^{(i)}\right\rangle .
$$

Equation (47) allows us to rewrite the eigenvalue as

$$
2 \lambda^{(i)}\left(H_{\alpha}\right)-\alpha_{i}\left(H_{\alpha}\right)=-\sum_{j \neq i} a_{i j} \lambda^{(j)}\left(H_{\alpha}\right)
$$

Finally, to find the proportionality factor in Eq. (48), it is enough to compute the norm of $\left|\Psi^{(i)}\right\rangle$,

$$
\left\langle\Psi^{(i)} \mid \Psi^{(i)}\right\rangle=\lambda^{(i)}\left(H_{\alpha_{i}}\right)=\left(\alpha_{i}, \alpha_{i}\right) .
$$

This ends the proof of the first part of the proposition. 
2) The second statement is obvious.

3) The third follows from the relations

$$
\begin{aligned}
& \operatorname{coth}\left(\pi \operatorname{ad}_{1} P_{0}\right)\left(r^{+}+r^{-}\right)=2 \sum_{\alpha \text { positive }} \operatorname{coth}\left(\pi \alpha\left(P_{0}\right)\right)\left[E_{\alpha} \otimes E_{-\alpha}+E_{-\alpha} \otimes E_{\alpha}\right], \\
& \operatorname{coth}\left(\pi \operatorname{ad}_{2} P_{0}\right)\left(r^{+}+r^{-}\right)=-2 \sum_{\alpha \text { positive }} \operatorname{coth}\left(\pi \alpha\left(P_{0}\right)\right)\left[E_{\alpha} \otimes E_{-\alpha}+E_{-\alpha} \otimes E_{\alpha}\right] .
\end{aligned}
$$

\section{Conformal Properties of the Field $\eta(x)$ and $\psi(x)$}

Recall that the energy momentum tensor reads

$$
\mathscr{U}=\frac{1}{2}(P, P)+\left(H_{\rho}, P^{\prime}\right) .
$$

For any periodic function $f$ we define

$$
f(\mathscr{U})=\int_{0}^{2 \pi} d x f(x) \mathscr{U}(x) .
$$

It is straightforward to show that

$$
\{f(\mathscr{U}), P(x)\}=-\partial(f(x) P(x))+\partial^{2} f(x) H_{\rho} .
$$

Using the same techniques as the ones developed in this paper one can show

\section{Proposition 11.1.}

$$
\begin{aligned}
\left\{f(\mathscr{U}), Q_{+}(x)\right\} & =-f(x) Q_{+}^{\prime}(x)+f^{\prime}(x) H_{\rho} Q_{+}(x)+Q_{+}(x)\left[f(0) Q_{+}^{\prime}(0)-f^{\prime}(0) H_{\rho}\right], \\
\{f(\mathscr{U}), S\} & =\left[S, f(0) Q_{+}^{\prime}(0)-f^{\prime}(0) H_{\rho}\right], \\
\{f(\mathscr{U}), g\} g^{-1} & =f(0)\left[g Q_{+}^{\prime}(0) g^{-1}-P(0)\right]-f^{\prime}(0)\left[g H_{\rho} g^{-1}-H_{\rho}\right] .
\end{aligned}
$$

From this we immediately obtain

$$
\begin{aligned}
\left\{f(\mathscr{U}), \eta^{(r)}(x)\right\}= & -\left[f(x) \partial-\lambda_{\max }^{(r)}\left(H_{\rho}\right) f^{\prime}(x)\right] \eta^{(r)}(x) \\
& +\eta^{(r)}(x)\left[f(0) P(0)-f^{\prime}(0) H_{\rho}\right] .
\end{aligned}
$$

The first term in this formula shows that $\eta$ behaves almost as a conformal field of weight $\lambda_{\max }^{(r)}\left(H_{\rho}\right)$.

To get a covariant object we define

$$
\psi(x)=\eta(x) \rho_{+},
$$

where $\rho_{+}$is introduced in Eq. (24). Using that

$$
\begin{aligned}
& \left\{f(\mathscr{U}), K_{+}\right\}=\left[f(0) P(0)-f^{\prime}(0) H_{\rho}\right]-\frac{1}{2 \pi} \int_{0}^{2 \pi} d x f(x) P(x), \\
& \left\{f(\mathscr{U}), \theta_{+}\right\}=-\frac{1}{2 \pi} \int_{0}^{2 \pi} d x f(x) P(x) \theta_{+},
\end{aligned}
$$

we find

$$
\left\{f(\mathscr{U}), \psi^{(r)}(x)\right\}=-\left[f(x) \partial-\lambda_{\max }^{(r)}\left(H_{\rho}\right) f^{\prime}(x)\right] \psi^{(r)}(x),
$$

which shows that $\psi^{(r)}(x)$ is a covariant operator. A similar analysis holds for $\bar{\psi}$. 


\section{The $\psi(x), \bar{\psi}(x)$ Basis}

The covariant basis $\psi, \bar{\psi}$ enjoys the following additional properties:

1) It has diagonal monodromy.

2) It has a vanishing Poisson brackets

$$
\{\psi(x), \bar{\psi}(y)\}=0
$$

3) The exchange algebra reads

\section{Proposition 12.1.}

$$
\begin{aligned}
\{\psi(x) \otimes, \psi(y)\}= & -\frac{1}{4} \psi(x) \otimes \psi(y)\left(r^{+}-r^{-}\right) \varepsilon(x-y) \\
& +\frac{1}{4} \psi(x) \otimes \psi(y)\left[\operatorname{coth}\left(\pi \operatorname{ad}_{1} P_{0}\right)\left(r^{+}-\sum_{i} H_{i} \otimes H_{i}\right)\right. \\
& \left.+\operatorname{coth}\left(\pi \operatorname{ad}_{2} P_{0}\right)\left(r^{-}+\sum_{i} H_{i} \otimes H_{i}\right)\right], \\
\{\bar{\psi}(x) \otimes, \bar{\psi}(y)\}= & \frac{1}{4}\left(r^{+}-r^{-}\right) \varepsilon(x-y) \bar{\psi}(x) \otimes \bar{\psi}(y) \\
& +\frac{1}{4}\left[\operatorname{coth}\left(\pi \operatorname{ad}_{1} P_{0}\right)\left(r^{-}+\sum_{i} H_{i} \otimes H_{i}\right)\right. \\
& \left.+\operatorname{coth}\left(\pi \operatorname{ad}_{2} P_{0}\right)\left(r^{+}-\sum_{i} H_{i} \otimes H_{i}\right)\right] \bar{\psi}(x) \otimes \bar{\psi}(y) .
\end{aligned}
$$

Proof. It is a straightforward consequence of the previous results.

These relations are the same as the ones obtained in [11].

In this basis the reconstruction theorem takes the form

\section{Theorem 12.2.}

1) The field

$$
e^{-\lambda_{\max }^{(r)}(\Phi)}=\psi^{(r)} \bar{\psi}^{(r)}
$$

is a solution to the equation of motions.

2) It admits a restriction to the original phase space, i.e.

$$
\left\{e^{-\lambda_{\max }^{(r)}(\Phi)}, P_{0}-\bar{P}_{0}\right\}=0 .
$$

3) The restriction of $e^{-\lambda_{\max }^{(r)}(\Phi)}$ is periodic.

4) This restriction is local.

Proof. It is straightforward.

It is evident that there are infinitely many choices of bases, each one of them having its own virtue depending on the problem one deals with $[12-15,18]$. A noticeable merit of our construction is that it works uniformly for any simple Lie 
algebra, and lends itself naturally to the extension to the affine Lie algebra case [9] and to the supersymmetric case as well [10].

\section{Appendix: Diagonalization}

We have determined in the text the Poisson brackets of the matrix $g$ which diagonalizes the monodromy matrix $S$,

$$
S=g^{-1} k g
$$

where

$$
k=e^{2 \pi P_{0}} .
$$

We stress again that for this we did not need the explicit form of $g$. In this appendix we construct explicitly the matrix $g$ for the $A_{n}$ case. One way to do this is to perform a gauge transformation on the Drinfeld-Sokolov linear system. Set

$$
\begin{aligned}
A & =P-\mathscr{E}_{+}, \\
(\partial-A) v & =0 .
\end{aligned}
$$

Performing a gauge transformation means

$$
\begin{aligned}
{ }^{g} v & =g \cdot v, \\
\left(\partial-{ }^{g} A\right)^{g} v & =0, \\
{ }^{g} A & =\partial g \cdot g^{-1}+g A g^{-1} .
\end{aligned}
$$

The transport matrix is defined by

$$
{ }^{g} v(x)={ }^{g} Q_{+}(x)^{g} v(0),
$$

so that

$$
Q_{+}(x)=g^{-1}(x)^{g} Q_{+}(x) g(0) .
$$

Setting $x=2 \pi$ we get

$$
S=Q_{+}(2 \pi)=g^{-1}(2 \pi)^{g} Q_{+}(2 \pi) g(0) .
$$

The diagonalization of $S$ is equivalent to finding a periodic gauge transformation such that ${ }^{g} A$ is diagonal. So we will look for a triangular $g$ such that

$$
{ }^{g} A=P
$$

or

$$
\partial g \cdot g^{-1}+g\left(P-\mathscr{E}_{+}\right) g^{-1}=P
$$

together with the boundary condition

$$
g(2 \pi)=g(0)
$$

Proposition 13.1. Equation (55) admits a unique solution which is periodic and strictly upper triangular.

Proof. Rewrite Eq. (55) in the form

$$
\partial g+g\left(P-\mathscr{E}_{+}\right)-P g=0 .
$$


Its general solution is

$$
g(x)=e^{K_{+}(x)} g(0) Q_{+}^{-1}(x) .
$$

We have

$$
g(x+2 \pi)=e^{K+(x)} k g(0) S^{-1} Q_{+}^{-1}(x) .
$$

Imposing that $g(x)$ is periodic gives

or

$$
g(0)=k g(0) S^{-1}
$$

$$
S=g^{-1}(0) k g(0) \text {. }
$$

This last equation has a unique strictly upper triangular solution $g(0)$. We see here again that finding the periodic solution of Eq. (55) is equivalent to the diagonalization of $S$.

13.1. The Case $A_{n}$. We will construct explicitly the solution of Eq. (56) in the case $s l(n+1)$. Let $\alpha_{1}, \alpha_{2}, \ldots, \alpha_{n}$ be the simple roots. The positive roots are

$$
\alpha_{i j}=\alpha_{i}+\alpha_{i+1}+\cdots+\alpha_{j}
$$

and there is no gap in the integers between $i$ and $j$.

We are going to find a gauge transformation $g_{1}$ such that

$$
{ }^{g_{1}} A=P-\sum_{i=2}^{n} E_{\alpha_{i}},
$$

i.e. ${ }^{g_{1}} A$ is analogous to $A$ but $\alpha_{1}$ does not appear any more. Repeating the construction $n$ times and setting

we will have

$$
g=g_{n} g_{n-1} \cdots g_{2} g_{1},
$$

$$
{ }^{g} A=P
$$

as desired. We first prove a preliminary result

Proposition 13.2. Let $X_{\beta}$ be a periodic function. The periodic solution of the differential equation

is

$$
\partial X_{\alpha}-\alpha(P) X_{\alpha}=X_{\beta}
$$

$$
X_{\alpha}(x)=Y_{\alpha}(x) \int_{0}^{2 \pi} d y k_{\alpha}(x, y) X_{\beta}(y) Y_{\alpha}^{-1}(y),
$$

where

$$
Y_{\alpha}(x)=\exp \left\{\int_{0}^{x} d y \alpha(P)\right\}
$$

is the solution of the homogeneous equation, and $k_{\alpha}(x, y)$ is the kernel

$$
k_{\alpha}(x, y)=\theta(x-y)-\frac{1}{1-e^{-2 \pi \alpha\left(P_{0}\right)}} .
$$

Proof. It is straightforward. 
Proposition 13.3. Let

$$
g_{\alpha_{1}+\alpha_{2}+\cdots \alpha_{p}}=\exp \left\{X_{\alpha_{1}+\alpha_{2}+\cdots \alpha_{p}}\left[\cdots\left[\left[E_{\alpha_{1}}, E_{\alpha_{2}}\right], E_{\alpha_{3}}\right] \cdots E_{\alpha_{p}}\right]\right\},
$$

where

$$
\begin{aligned}
X_{\alpha_{1}+\alpha_{2}+\cdots \alpha_{p}}(x)= & Y_{\alpha_{1}+\alpha_{2}+\cdots \alpha_{p}}(x) \int_{0}^{2 \pi} d y_{p} \int_{0}^{2 \pi} d y_{p-1} \cdots \int_{0}^{2 \pi} d y_{1} \\
& \cdot k_{\alpha_{1}+\cdots+\alpha_{p}}\left(x, y_{p}\right) k_{\alpha_{1}+\cdots+\alpha_{p-1}}\left(y_{p}, y_{p-1}\right) \cdots k_{\alpha_{1}}\left(y_{2}, y_{1}\right) \\
& \cdot Y_{\alpha_{p}}^{-1}\left(y_{p}\right) \cdots Y_{\alpha_{1}}^{-1}\left(y_{1}\right) .
\end{aligned}
$$

Then setting

$$
g_{1}=g_{\alpha_{1}+\alpha_{2}}+\cdots \alpha_{n} g_{\alpha_{1}+\alpha_{2}}+\cdots \alpha_{n-1} \cdots g_{\alpha_{1}}
$$

we have

$$
{ }^{g_{1}} A=P-\sum_{i=2}^{n} E_{\alpha_{i}}
$$

Proof. Let

then

$$
g=g_{\alpha_{1}}=e^{X_{\alpha_{1}} E_{\alpha_{1}}}
$$

$$
{ }^{g} A=\partial X_{\alpha_{1}} E_{\alpha_{1}}+e^{\mathrm{ad} X_{\alpha_{1}} E_{\alpha_{1}}}\left(P-\mathscr{E}_{+}\right)
$$

Obviously

but also

$$
\left(\operatorname{ad} X_{\alpha_{1}} E_{\alpha_{1}}\right)^{2}(P)=0
$$

$$
\left(\operatorname{ad} X_{\alpha_{1}} E_{\alpha_{1}}\right)^{2}\left(\mathscr{E}_{+}\right)=0 .
$$

This is because in $\operatorname{sl}(n+1)$ there is no positive root of the form $2 \alpha_{1}+\cdots$. Thus

$$
{ }^{g} A=\partial X_{\alpha_{1}} E_{\alpha_{1}}+P-\mathscr{E}_{+}+\left[X_{\alpha_{1}} E_{\alpha_{1}}, P\right]-\left[X_{\alpha_{1}} E_{\alpha_{1}}, \mathscr{E}_{+}\right] .
$$

In $\operatorname{sl}(n+1)$ we have

$$
\left[E_{\alpha_{1}}, \mathscr{E}_{+}\right]=\left[E_{\alpha_{1}}, E_{\alpha_{2}}\right]
$$

So that finally

$$
{ }^{g} A=\left\{\partial X_{\alpha_{1}}-\alpha_{1}(P) X_{\alpha_{1}}-1\right\} E_{\alpha_{1}}+P-\sum_{i=2}^{n} E_{\alpha_{i}}-X_{\alpha_{1}}\left[E_{\alpha_{1}}, E_{\alpha_{2}}\right] .
$$

We choose $X_{\alpha_{1}}$ as the periodic solution of the equation

$$
\partial X_{\alpha_{1}}-\alpha_{1}(P) X_{\alpha_{1}}=1,
$$

that is to say

$$
X_{\alpha_{1}}(x)=Y_{\alpha_{1}}(x) \int_{0}^{2 \pi} d y k_{\alpha_{1}}(x, y) Y_{\alpha_{1}}^{-1}(y) .
$$

With this choice we have

$$
{ }^{g} A=P-\sum_{i=2}^{n} E_{\alpha_{1}}-X_{\alpha_{1}}\left[E_{\alpha_{1}}, E_{\alpha_{2}}\right]
$$


Next we set

and

$$
g_{\alpha_{1}+\alpha_{2}}=\exp \left\{X_{\alpha_{1}+\alpha_{2}}\left[E_{\alpha_{1}}, E_{\alpha_{2}}\right]\right\}
$$

We get

$$
g=g_{\alpha_{1}+\alpha_{2}} g_{\alpha_{1}} \text {. }
$$

$$
\begin{aligned}
{ }^{g} A= & \partial X_{\alpha_{1}+\alpha_{2}}\left[E_{\alpha_{1}}, E_{\alpha_{2}}\right]+P-\sum_{i=2}^{n} E_{\alpha_{i}} \\
& +X_{\alpha_{1}+\alpha_{2}}\left[\left[E_{\alpha_{1}}, E_{\alpha_{2}}\right], P\right]-X_{\alpha_{1}+\alpha_{2}}\left[\left[E_{\alpha_{1}}, E_{\alpha_{2}}\right], \sum_{i=2}^{n} E_{\alpha_{i}}\right]-X_{\alpha_{1}}\left[E_{\alpha_{1}}, E_{\alpha_{2}}\right]
\end{aligned}
$$

or

$$
\begin{aligned}
{ }^{g} A= & \left\{\partial X_{\alpha_{1}+\alpha_{2}}-\left(\alpha_{1}+\alpha_{2}\right)(P) X_{\alpha_{1}+\alpha_{2}}-X_{\alpha_{1}}\right\}\left[E_{\alpha_{1}}, E_{\alpha_{2}}\right] \\
& +P-\sum_{i=2}^{n} E_{\alpha_{i}}-X_{\alpha_{1}+\alpha_{2}}\left[\left[E_{\alpha_{1}}, E_{\alpha_{2}}\right], E_{\alpha_{3}}\right] .
\end{aligned}
$$

We choose $X_{\alpha_{1}+\alpha_{2}}$ as the periodic solution of

$$
\partial X_{\alpha_{1}+\alpha_{2}}-\left(\alpha_{1}+\alpha_{2}\right)(P) X_{\alpha_{1}+\alpha_{2}}=X_{\alpha_{1}}
$$

or explicitly

$$
X_{\alpha_{1}+\alpha_{2}}(x)=Y_{\alpha_{1}}(x) Y_{\alpha_{2}}(x) \int_{0}^{2 \pi} d y \int_{0}^{2 \pi} d z k_{\alpha_{1}+\alpha_{2}}(x, y) k_{\alpha_{1}}(y, z) Y_{\alpha_{2}}^{-1}(y) Y_{\alpha_{1}}^{-1}(z) .
$$

With this choice we have

$$
{ }^{g} A=P-\sum_{i=2}^{n} E_{\alpha_{i}}-X_{\alpha_{1}+\alpha_{2}}\left[\left[E_{\alpha_{1}}, E_{\alpha_{2}}\right], E_{\alpha_{3}}\right] .
$$

Clearly one can repeat the procedure and obtain in this way the announced result.

It is remarkable that the order of the factors in Eqs. $(57,58)$ is exactly the same as the one appearing in the universal quantum $R$-matrix of $\mathscr{U}_{q}\left(s l_{n}\right)$, as found by M. Rosso [16]. For other Lie algebras we expect that this order is the one obtained from the longest element of the Weyl group $[17,18]$.

\section{References}

1. Gervais, J. L., Neveu, A.: Nucl. Phys. B238, 125 (1984)

2. Babelon, O.: Phys. Lett. B215, 523 (1988)

3. Babelon, O., Bonora, L.: Conformal affine $s l_{2}$ Toda field theory, S.I.S.S.A 36 EP (Apr 90)

4. Leznov, A. N., Saveliev, M. V.: Lett. Math. Phys. 3, 489 (1979)

5. Drinfeld, V. G., Sokolov, V. V.: J. Sov. Math. 30, 1975 (1984)

6. Gervais, J. L., Bilal, A.: Nucl. Phys. B314, 646 (1989)

7. Olive, D., Turok, N.: Nucl. Phys. B257, 277 (1985); Nucl. Phys. B265, 469 (1986)

8. Jimbo, M.: Commun. Math. Phys. 102, 537 (1986)

9. Babelon, O., Bonora, L.: Proceeding of the Rapallo Conference, June 1990, SISSA preprint

10. Babelon, O., Toppan, F.: in preparation 
11. Balog, J., Dabrowski, L., Fehér, L.: Classical $r$-matrix and exchange algebra in WZNW and Toda theories, DIAS-STP-90-4

12. Faddeev, L. D.: On the exchange algebra for WZNW model, HU-TFT-89-56

13. Gervais, J. L.: The quantum group structure of $2 D$ gravity and minimal models, LPTENS $89 / 14$

14. Cremmer, E., Gervais, J. L.: The quantum group structure associated with non-linearly extended Virasoro algebras LPTENS 89/19

15. Alekseev, A., Shatashvili, S.: Quantum group and WZW models, EFI-89-67

16. Rosso, M.: Commun. Math. Phys. 124, 307 (1989)

17. Levendorskii, S. Z., Soibelman, Y. S.: Some applications of the quantum Weyl group. 1 The multiplicative formula for the universal $R$-matrix for simple Lie algebras, Preprint

18. Kirillov, A. N., Reshetikhin, N.: $q$-Weyl group and a multiplicative formula for universal $R$-matrices, Preprint

Communicated by N. Yu. Reshetikhin 
